\title{
Reification Fallacies and Inappropriate Totalities
}

\section{Nicholas Rescher University of Pittsburgh}

\begin{abstract}
As Russell's paradox of "the set of all sets that do not contain themselves" indicated long ago, matters go seriously amiss if one operates an ontology of unrestricted totalization. Some sort of restriction must be placed on such items as "the set of all sets that have the feature $F$ " or "the conjunction of all truths that have the feature $G$." But generally, logicians here introduce such formalized and complex devices as the theory of types or the doctrine of impredictivity. The present paper argues for the informal and elementary idea that the items invoked in a proper identification have themselves already been identified. Even as an explanation is not satisfactory that proceeds in terms of items that themselves require prior explanation, so the same holds with identification. And heed of this elementary idea suffices to sideline those otherwise paradoxical perplexities.
\end{abstract}

Résumé: Le paradoxe de Russell, a'ensemble regroupant tous les ensembles qui appartiennent à eux-mểmes", a démontré depuis longtemps que nos réflexions se désorientent si nous employons une ontologie de totalisation sans restriction. Une limite doit $s$ 'imposer sur des objets tels que al'ensemble de tous les ensembles qui ont la propriété $G$ » ou "la conjonction de toutes les vérités qui ont la propriété $\mathrm{G}$ \%. Les logiciens introduisent généralement des approches formelles et complexes comme la théorie des types ou la doctrine d'kimpredictivity». Je soutiens que l'idée non formelle et élémentaire que les objets invoqués dans une identification appropriée ont déjà eux-mêmes été identifiés. Une explication dont les termes eux-mêmes requierent une explication antérieure est insatisfaisante: il en va de même pour une identification. Une attention à cette idée élémentaire suffit pour écarter ces perplexités paradoxales.

Keywords: totality, identification, self-identification, sets, paradox, set theory paradox, Barber paradox, Russell, Russell's Paradox,Vicious Circle Principle, Kant's antinomies

\section{Improperly Totalized Wholes and Illicit Reification}

The salient message of this discussion is that some of the problems and difficulties that logicians generally seek to overcome by elaborate formal devices such as the theory of types can be averted by straightforward informal devices, namely the rejection of inappropriate anaphora, the mistake committed in communication when a back-reference treats an item yet to be specified as already identified.

The circumstance that questions can rest on inappropriate suppositions has far-reaching implications. In particular, it means that there are-or can be-items 
of discussion that are no more than pseudo-realities which fail to give rise to meaningful questions and do not qualify as a viable subject of further inquiry. And it is noteworthy that various sorts of putative totalities constitute a case in point.

For ostensive identification we need a something descriptive, namely a sorted classifier. I do not identify by simply "this (pointing)" without adding "table" or "table cloth" or "dark speck on the table cloth" or the like. And there is, in principle, no limit to the number of sortals that can be added to specify what is at issue with an ostensive act of "this (pointing)."

Now a physical particular, as Aristotle rightly insisted, is something comfortable, as a tode $t i$ that is simply a possible target for a "this (pointing)." And we cannot count what we cannot identify. Hence we cannot count what is altogether nondescript-or otherwise unspecified particular, thing, object, item, similar indefinite. It makes sense to ask how many books or flies are in the room but not how may things or items are in the room. To count particulars, we must first sortalize them.

And what we cannot count we cannot totalize either. There is no total for objects (items, things, particulars, and individuals) in the abstract. We can only have totals where we have to do with descriptive kinds of things. To do meaningful totalization we require meaningful identification and this in turn means that we require descriptive sortalization. Totalities in sum are correlative with type-coordinated kinds of things.

Moreover, the fact that we cannot totalize what we cannot count and that we must count what we cannot identify means that the items at issue in a totalization must have an identification that is accessible to us independently of and prior to the process of totalization itself.

To introduce something into deliberation or discussion as a meaningful item of consideration we have to proceed by way of expository definitions or explanatory specifications that employ only discursive materials that themselves have already been introduced - at any rate apart from whatever may qualify as a primitive on the basis of being so self-evident as to require no further introduction. And, above all, the identification of something can be achieved satisfactorily only through reference to items that have already been identified themselves. It does us little good to be told that something we seek is sited next to something else whose location we do not know.

Thus consider the following identification-purporting specifications:

- the individual in question is the first child of the mother who is this person's female parent.

- the position in question is the one to the left of that position which is to its immediate right.

- the woman in question is the wife of the man who is her husband. Although there is some description going on in all such cases, there is certainly no question of any effective introductory identification. And when God responded to 
Moses question "Who are you?" with the statement "I am the one that I am" he was not being particularly helpful-though this very fact gave an appropriate response by redirection: "There is really nothing I can say in your language or convey by your concepts that would be adequate in answering your question."

To adopt the identifying specification

$$
\begin{aligned}
X= & \text { the item that bears such-and-such a relationship to } X \text { (or "is in- } \\
& \text { volved in such-and-such a way with } X " \text { ) }
\end{aligned}
$$

is identificatorily unavailing. In all cases of this sort the formula's anaphoric backreference to the item in question itself is a factor that supposes an already effected identification. All of these formulations fail as identifications because they require a prior item-identification which (by hypothesis) is unavailable in view of the identification processes that are being embarked upon. In each case the purported identification manifestly presupposes a yet-unavailable answer to the identificatory question that is being addressed.

Here, as elsewhere, what that answer is depends on just exactly what the question at issue happens to be. If the question is one of introductory individuationof identificatory specification-then referential self-involvement is inappropriate because the "self" at issue is not yet in hand. On the other hand, if it is only a matter of the description or characterization of some pre-identified item, then selfinvolvement is harmless because the "self" at issue has already been specified. As long as the self-reference at issue is merely a matter of dealing with something that is already specified, there is no problem. But whenever we are dealing with the introduction of items by way of an initial specification or definition, then it is clear that the availability of these items cannot be appropriately assumed at this stage.' Self-referential item-specifications and identifications are by their very nature ineffectual because until the item supposedly at issue has been properly identified, there is nothing to discuss.

Now what holds here of identification also holds of any meaningful totalization. Totalization as such is harmless enough. The totality of $X^{\prime} s$ is the putative item that includes all of the $X$ 's - that is, contains or embraces them in whatever way is appropriate to the sort of thing at issue. Thus the totality of letters of the alphabet is the alphabet as such, the totality of colors is the entire color spectrum, or the totality of plants is the entire kingdom of flora. However, self-referential totalization is by nature an illicit process because identification or specification of a totality is meaningfully practicable only when the item being totalized has already been identified antecedently to the totalization process itself.

Thus to define a certain something $(Z)$ by way of a formula of the format

$Z=$ the total of a group of items including $Z$ itself

is also unavailing. It is one thing - a harmless one-to be given a total and thereupon to learn that it is duly self-inclusive-a "fractal" rectangular pattern, say, within each of whose four quadrants this very pattern is repeated all over again. But it is something very different-and altogether vitiating-to attempt to intro- 
duce (define, identify, specify) a totality in a way that demands or presupposes that this putative item is already available through previous introduction.

Meaningful totalizations cannot involve an anaphoric back-reference that is self-referential; such reference must proceed with respect to items that have an antecedently established identity. ${ }^{2}$ And we therefore cannot appropriately effect a totalization whenever its purported product is itself a candidate for inclusion among the items being totalized. Only when its constituents are antecedently identified can we have a meaningfully defined totality that is to be comprised of "them." And we are certainly not entitled to presuppose the prior specification of a (totalized) item whose specification is only now in progress

Perplexity is thus bound to arise when there is homogenous totalization, that is, when the items being totalized are of the same sortal type (thing-kind) as the totality that is supposedly at issue. Accordingly, there is a decisive difference between the identificatory specification of "The set of all sets that include less then five members," which is innocuous since this set certainly cannot include itself, and the specification of "The set of all sets that include more than five members" which-were it to exist-would have to include itself. The homogeneous totalization at issue accordingly represents a putative item that the formula at issue fails to specify in a meaningful way. Self-referential identifications are by nature inappropriate, and we must accordingly place a conceptual embargo on self-inclusively characterized totalities.

This occurs in such cases as when the totality of sets is supposed to be a set that includes all sets, the totality of regions a region that includes all regions, or the totality of timespans a timespan that includes all timespans; and the like. All such attempts at identification are uniformly unsuccessful because no meaningful item-introduction is effected when the totality that is being introduced is itself one of the items being totalized: when it is, so to speak, one of its own members (elements, constituents, components, or the like). For then it is implicitly supposed (via the presence of that "all") that the total being introduced is itself already available as an item for potential inclusion in something. And the difficulty that arises with homogenous totalization is clearly the problem of self-inclusion. The membership of a meaningfully identified totality must have a pre-established identity: those envisioned members must be identified prior to and thereby independently of the totalization that is in progress.

Just as the object of a meaningful item-specification must have a pre-established identity independent of that specification itself, so also with objects that are being gathered together in an item-totalization. But when the totality supposedly at issue is homogenous with the items being totalized, then of course we would have to have it that the totalization at issue is vitiated. It is, clearly, inappropriate to offer a specification based on the supposition that the very item at issue in this specification has already been specified. For meaningful (proper) identification we cannot presuppose the availability as input into the identification process the very item itself that is supposed to be the output of that process. The illegitimacy of self- 
involving specifications roots in the fact that self-reference presupposes that the item in question is already available as a meaningful unit of discussion. ${ }^{3}$

There can, of course, be comprehensive totals and they can be comprehensive in rather different ways. A whole is $T$-maximal in the sense of Nicholas of Cusa ${ }^{4}$ if it itself has $T$-parts but is itself a $T$-part of nothing further (i.e., of nothing other than possibly itself). Thus a human body is biologically maximal since it has biological parts (e.g., cells) but is not itself a biological part of something larger. A book, on the other hand, is not literally maximal for while it has literary parts (chapters) it is itself a literary part of something larger (a series or even a library). Again the cosmos-as-a-whole is - obviously - physically maximal. But totalization is a means for introducing something new into the arena of discussion. And such introductions can be self-referential only at the cost of self-vitiation.

Let us adopt the notation $\langle C\rangle$ to abbreviate "the totality that embraces (that is, $C$-relevantly 'embraces') all of the $C$-type items." Thus, for example, if $C(x)=$ " $x$ is a letter of the (Roman) alphabet," then $\langle C\rangle$ is the totality of letters of the (Roman) alphabet, which is to say it is that alphabet itself. If $C(x)=$ " $x$ is a cat" then $\langle\mathrm{C}\rangle$ is the totality of cats, the entire genus Felix that includes all the felines there are. Or again, if $C(x)=$ " $x$ is a color" then $\langle C>$ is the totality of colors, which is to say it is the whole color spectrum. On the basis of the preceding deliberations it is clear that whenever the totality $<C>$ that is presumably being defined is "itself" seen as something that meets condition $C$-which is emphatically not the case with any of the preceding examples - then the totalization process in question goes awry since it is now unable to realize a well-defined result. A purported item that is not subject to a discussion-introducing identification in terms of reference independent of itself has simply not been introduced meaningfully into the discussion at all.

Examples of putatively totalized wholes that violate the proscription of selfpresupposition include the following pseudo-totalities:

The supertruth understood as the truth that conjoins all truths.

The cosmic fact about the world understood as a fact that encompasses all facts about the world.

The megaset understood as the set that includes all sets.

Eternity understood as the timespan that contains all timespans.

The panexplanation understood as the explanation that encompasses all explanations (that explains everything).

The protocause understood as the cause that causes all causes.

The mega-story understood as the story that encompasses all stories.

The superadjective understood as the adjective that applies to all adjectives.

The world-all (Weltall) understood as the physical region that includes all physical regions. 
The omni-cause understood as the cause that causes literally everything that is real.

In each case we have a putative item that is identified by means of a common format: "The $C$-type item that "embraces' all $C$-type items." And this clearly presupposes not only that there indeed is (a unique) something that "embraces" all $C$ type items, but that this something is itself of type $C$. Throughout, the proscription of self-involving totalization is thus violated. The boundaries of that purported total at issue are not something that can be settled unproblematically until we have determined the viability of that total itself as an item of its purported kind.

There yet remains the prospect of objecting: "But if it is defined as an $X$, if its $X$-hood is something that is explicitly specified in its definition, then surely it's got to be an $X$ !" This plausible protest does not, however, hold water. As the longstanding critique of the Ontological Argument shows, substantive questions cannot be settled by definitional fiat. (This is a cardinal principle of rational inquiry.) Calling something an $X$ does not mean that this is an actual item that is actually an $X$. Thus "the integer that is larger than any other" is a formula that purports to specify an integer but actually fails to do so, seeing that there is no integer larger than all others. And similarly to call a supertruth a truth or a megaset a set does not mean that this is actually so-that there really is a set or a truth that answers to the specification at issue. For that item may simply not exist as such. The assumption that something exists under a certain description-for example, as the set of all sets that do not include themselves-may well be false. Anything that answers to this description-whatever it might be-cannot be a set. That supposed specification simply fails to identify.

To be sure, hypostatization (item-introduction) is one thing and mere description (of a pre-identified item) something else again. Once we have an item at hand (say 2 as "the smallest prime") one can describe it in self-inclusive terms (as "the prime that is not undersized by any prime"). But we cannot properly introduce it in this way until the issue of existence has been (independently) resolved. There is thus a crucial disanalogy between identifying something on the one hand and describing it on the other. We can only consider "the thing identified" once some "identification of the thing" has been given: any possible entertainment of the former (the thing identified) is contingent upon the latter (the identification of the thing). By contrast, nothing whatever about the describability of something rests upon the description of this thing. The hypothetical removal of its identification creates problems for discourse about something in view in a way that the hypothetical removal of its description would not.

Accordingly, there is no problem about (say) characterizing a pre-identified being (i.e., God) as that which is "the ultimate reason of all being" or again as that which "self-caused-causa sui-the cause of itself." Describing an already individuated God by such formulas could in theory qualify as perfectly meaningful. But we nevertheless cannot identify God by a decision-introducing characterization as "the reason-for-being of all reasons-for-being" or as "that being which is 
the cause of all being itself included." For when seen as an identifying specification self-reference must be rejected as counter-productively vitiating. We can certainly say - truly and meaningfully - of some pre-identified item that "it is the result of the cause that produced it." But we cannot use this sort of formula when the initial identification of the item is at issue ("the item that is to be at issue is the product of its cause"). For here nothing is as yet available to serve as referential for that ultimate anaphoric back-reference. Unlike inappropriately described items, inappropriately introduced items are no more than illusions.

A significant lesson emerges. Not only are there pseudo-questions like "Have you stopped cheating on your taxes?" that should not be asked (because they rest on inappropriate suppositions) but there are pseudo-identifications that should not be considered (investigated, taken seriously) because their very conception is flawed in that it rests on the erroneous presupposition of a particular answer to an inappropriate question. For successful identification requires and presupposes an affirmative answer to the question: "Have all the items being referred to already been identified effectively?"

Let us be somewhat more explicit about the ramifications of this state of affairs.

\section{The Route to Paradox}

All contexts of rational assertion and deliberation are governed by an at least tacit supposition that its terms of reference are meaningful. Should this presupposition prove to be unwarranted, the chasm of paradox yawns wide-open before us. This is illustrated by a conundrum that has received much attention is The Barber Paradox. ${ }^{6}$ It is based on the following riddle:

A certain village has a barber whose practice it is to shave all the adult male villagers who do not shave themselves. He himself, of course, is an adult male who lives in the village. Does he or does he not shave himself?

The evident paradox here is that if he is a self-shaver, then (by hypothesis) our barber does not shave himself, while if he is not a self-shaver, then (by hypothesis) he does shave himself. Either way we are in difficulty.

Putting it more explicitly, our barber $B$ is (by specificatory hypothesis) such that:

$(\forall x)[S(B, x)$ iff $\sim S(x, x)]$.

And when the $x$ at issue is instantiated as $B$ himself, this yields:

$S(B, B)$ iff $\sim S(B, B)$.

This result plunges us into paradox, seeing that we now can maintain neither $S(B$, $B)$ nor $\sim S(B, B)$. It seems that consistency is beyond our reach here.

The following theses constitute the aporetic cluster that defines paradox: 
(1) There is-or can be-a barber who answers to the specifications of the narrative.

(2) This barber at issue in the narrative (like any other) either does or does not shave himself, but not both.

(3) If the barber shaves himself then, by the narrative's stipulation he is not someone who shaves himself.

(4) If the barber does not shave himself then, by the narrative's stipula tion he does shave himself.

(5) Thus either way a contradiction ensues.

The optimal point at which to break the chain of inconsistency here is clearly at its very start with that hypothetical barber himself. For there is not and cannot be a barber who answers to the specified conditions. The barber paradox thus is vitiated from the very outset, by being predicated on the supposition of a barber who cannot possibly be. That purported introduction of a barber upon the stage of discussion misfires-it fails to introduce. And what holds for inappropriate identifications also holds for inappropriate totalizations.

With such self-encompassment in view we can now project. Specifically, consider what might be characterized as the $X$-totalization of self-nonencompassing $X$ 's

the $X$ of all those $X$ 's that do not $X$-wise encompass themselves (the set of all those sets that do not set-include themselves, the shaver of all these shavers who do not shave themselves, or the like).

It is clear that any such totalization is always paradoxical. Thus consider the container (set) of all containers (sets) that do not contain (set-include) themselves, the list of all lists that do not list themselves, the picture of all pictures that do not depict themselves, etc. In each case we have a totality $Z$ of such a sort that :

\section{$Z$ enc $X$ iff $\sim(X$ enc $X)$.}

But now whenever $Z$ itself belongs to the range of the $X^{\prime} s$ at issue we shall arrive at the upshot:

$Z$ enc $Z$ iff $\sim(Z$ enc $Z)$.

And now we confront the question: Does this totalitarian $X$-item $Z$ encompass itself $X$-wise or not: does that set include itself, that list list itself, that picture picture itself, etc. And paradox occurs immediately because in virtue of its very definition that totality of self-exclusive $X^{\prime} \mathrm{s}$ is itself an $X$ that neither can nor cannot encompass itself. The problem, of course, is that the presupposition that such a negatively totalitarian $Z$ does or can exist is simply false. The item purported to be an instance of the kind at issue (a set, a list, etc.) just does not exist at all: to call it such does not make it such.

The preceding difficulties relate to the mis-identification of items purported to be of a type to which they could not possibly belong in view of the way in which that identification is presented. Throughout this range of cases, we have paradox 
of inappropriate presupposition that incorrectly assumes that something is appropriately identified by a specification which actually does not and cannot succeed. Any body of reasoning or argumentation presumes, explicitly or tacitly, that its propositions are meaningful and thus that its relevant terms are well-defined. And when terms become unraveled on the basis of an inappropriate totalitarian selfinvolvement, this crucial presupposition is falsified and the paradox dissolved. When the charge of illicit totalization can be made to stick, it is a highly effective paradox-buster.

\section{The Root of the Problem}

Recall that the identification operator $(x) P x$ so functions that (1) it is undefined unless there is just exactly one specifiable item that has the property $P$, and (2) when there is just exactly one such item, then $(L x) P x$ is (identical with) that item. And here saying that $(u x) P x$ is properly defined—symbolically $E !(u x) P x$-is to say that it represents a unique item belonging to the range to our universal quantifier $\forall$. Thus understood, $(v x) P x$ will be defined only when $P$ plainly and unequivocally applies to a single object. And it remains undefined whenever:

(i) $P$ is uninstantiated: it applies to nothing at all ( $P x$ is always false).

(ii) $P$ is multiply instantiated: it applies to several distinct objects ( $P x$ is true for several values of $x$ ).

(iii) $P$ is problematic: it is unclear where it applies ( $P x$ is not clearly determinable for some potential values of $x$ ).

Now let $\mu$ represent membership in a collectivity (of some, i.e., any sort). Then we may define:

(T) $(\tau x) P x={ }_{\mathrm{df}}(1 y) \forall x(x \mu y \equiv P x)$.

Accordingly, $(\tau x) P x$-or $\langle P\rangle$ as symbolized above-is the ( $\mu$-correlative) collectivity of all the items that have the property $P$. Specifically when $\mu$ is $\in$ the set membership of the mathematical theory of sets, then

$$
(\tau x) P x={ }_{\mathrm{df}}(y) \forall x(x \in y \equiv P x) .
$$

And it should be emphasized that $(u x) P x$ is undefined whenever-as in this $\in$ oriented case, thanks to Georg Cantor's Power Theorem-there fails to be just exactly one single unique $x$ such that $P x$ obtains. This of course means that ( $\tau x) P x$ will also be undefined in analogous circumstances. In particular, when $P$ is an "unsuitable" predicate- -say because it is equivocal or ill-defined-then $(\tau x) P x$ will remain undefined. On this basis, a properly-defined total exists whenever-but only whenever - the individuals that have the property at issue constitute a unique, well-defined set. The unfeasibility of totalization in unsuitable conditions is now not a distinctive and characteristic phenomenon but simply a natural consequence of the definitional specification of $\tau$ in terms of $\imath$.

Accordingly, the principle at issue with the present approach is a limitation on the introduction (or specification or definition) of totalities. It reads: 
Illicit Totalization Principle (ITP): The attempted specification of a total of items of a certain type is not meaningful unless-when itself is purported of the type at issue-the question of its self-membership can be resolved unproblematically in its favor. To be meaningfully and viably introduced into the discussion (that is, adequately specified, identified, defined) a totality (collection or whole) must not be purported in its introducing definition to include itself.

If this principle is violated, then we certainly run the risk of paradox. For note that this principle so functions as to preclude all of the following item specifications:

- the set of all sets [including that set itself] (Cantor's Paradox);

- the set of all sets satisfying a certain condition whose satisfaction by the set itself is unresolved (Curry's Paradox).

Given the inappropriateness of the item-specification at issue, no separate mechanism need be adopted to avert those associated paradoxes. The issue of identification-legitimacy is a powerful tool that suffices our needs here. No separate more elaborate mechanism is required to avert paradox in these indicated cases.

And so, given definition ( $T$ ) it follows theorematically that totalization hinges on the nature of the factor at issue: unsuitable predicates demonstrably do not totalize. Accordingly, no special machinery along the lines of a theory of set-types need be adopted to resolve the paradoxes posed by "illicit totalities": those paradoxes simply do not arise because these problematic totalities are not defined.

\section{Illicit Totalities}

There is no problem about the idea of a list of lists or a bibliography of bibliographies; such things exist in plenty and are altogether unproblematic. In principle we can even contemplate a list of all lists or a bibliography of all bibliographies: a complete list of lists or a complete bibliography of bibliographies. But what of the idea of:

- a complete list of all incomplete lists;

- a complete bibliography of all incomplete bibliographies.

If such a work includes itself, then it is ipso facto ineligible for self-inclusion. But if it does not include itself, then it is eligible for self-inclusion and for that very reason fails to answer to its own specification. What this clearly means is that the very idea of such works is self-contradictory, and the work supposedly at issue cannot possibly exist. A list of incomplete lists or a bibliography of incomplete bibliographies represent practical ideas, but totalization is inherently impracticable here. For what we have here is a fallacy of erroneous presupposition analogous with the erroneous presuppositional "loading" at issue in the question "Have you stopped cheating on your taxes?"

For the fact of the matter is that not every otherwise plausible item-specification can be totalized. Consider such specifications as: 
- a list of self-omitting lists

- a bibliography of self-omitting bibliographies

- an inventory of self-omitting inventories

- a set of self-omitting sets

- a discussion of self-ignoring discussions

So far, so good. However, otherwise meaningful item-specifications cannot be totalized. The very idea of a complete list of all self-omitting lists (or bibliographies, etc.) is absurd. What we have here throughout is a problem of one uniform phenomenon: the illicit totalization of the item at issue.

But just why should it be that we cannot totalize on those otherwise practicable and unproblematic characterizations? This question needs and deserves a detailed scrutiny.

Observe that the Illicit Totalization Principle is not one that governs the membership of totalities - let alone their existence as such - but one that merely addresses the proprieties of how totalities can meaningfully be introduced upon the stage of discussion and consideration. It deals with issues of communicative procedure, not issues of existence as such, and is accordingly a principle of logical grammar rather than one of ontology.

The principal lesson of this discussion, however, is that recourse to elaborate formal devices for paradox evasion such as the theory of types can in various cases be averted by the employment of a very straightforward and far less elaborate informal device, namely the eminently plausible proscription of identificatory self-reference.

\section{A Russellian Digression}

What is at issue here is closely allied to-but yet not identical with - a principle that Bertrand Russell took over from the French mathematician Henri Poincaré and which, following him, Russell characterized as the

Vicious Circle Principle (VCP): No collection (whole or totality) can contain members that are defined in terms of itself: specifically, no collection can ever be a constitutive part of itself. ${ }^{8}$

As it stands, this is clearly a limitation upon the constitution of totalities - and a very strong limitation at that. To quote Russell: "Whatever involves all of a collection must not itself be one of the collection." Saying that such a "collection" "has no total" is to say that it does not exist as a collection. What we have here is a restriction on the sorts of collections that can exist-that is, upon how authentic collections can validly be constituted.

And Russell's approach has a serious drawback. As he saw it, we must not stake claims about "all propositions" or about "all properties" because such locutions involve violations of his Vicious Circle Principle. ${ }^{10}$ However, he was so intent on barring illicit self-involvement that he insisted on barring self-involvement in 
general. In seeking to eliminate paradox, Russell also similarly dismisses a great deal of innocuous stuff as well-including such tautologically harmless universalizations as "All meaningful propositions make an assertion of some sort" or "All properties can be attributes to some sort of object," or "All objects can be members of collections." Someone prepared to subject logic to the requirements of common sense might well see this consequence alone as vitiating his version of the Vicious Circle Principle on grounds of throwing out the baby with the bath water.

To recapitulate: while the Russellian Vicious Circle Principle is ontological in its nature, the presently contemplated Illicit Totalization Principle is merely communicative or semantical-it only deals with the expository proprieties of how items of discussion can meaningfully be placed upon the agenda of consideration. The one principle deals with matters of actual existence, the other merely with matters of appropriate specification. Against this background it should be observed that the bearing of our present analysis of the implications of "vicious circularity" is purely terminological. It pivots upon showing that various putatively identifactory specifications will not succeed in placing a certain putative item upon the stage of consideration. And the rationale at issue is simply that a particular individuative presupposition is violated by the use of such an expression, thereby failing to enable the expression in question successfully to establish its intended reference.

An instructive lesson thus emerges. Russell sought an existence-negatory rationale for rejecting certain totalities. And he thought to find this in a conception of inappropriate existence claims based on a complex theory of types. But in fact a much simpler rationale is available. It lies in construing totalization $(\tau)$ in terms of definite description ( $l$ ) as per definition $(T)$ above. For then the fact that definite descriptions fail to identify in certain conditions automatically provides a rationale for seeing totalization in the same light. What is now at issue with improper totals is a problem of identification rather than one of being or existence-an epistemic rather than ontological principle.

And it is fortunate that the present approach is far less drastic. What is wrong with the "set of all sets that do not contain themselves" is-on the present account-not its excessive inclusiveness as a presumptive set but rather the anaphorically self-invoking back-reference-via the expression "themselves"that is at work in its formulation. In short, what the present approach proscribes is not self-referential impredicativity in general, but only its presence in identificatory contexts. (There is nothing wrong with a descriptive report along the lines that some pre-identified set "is a set that does (or does not) contain itself" as such.)

The proscription of impredicative self-involvement in item-specification is thus to all appearances a sensible policy. But now in taking this line in the present context we place a restriction not on existing totalities as such, but on the sorts of predications that can meaningfully be employed in the course of identifying totalities so as to introduce them into the discussion. 
To avoid paradoxes, then, we need not reject the existence of certain kinds of thing; it suffices to reject the appropriateness of certain particular ways of talking about things. The inappropriateness at issue turns on answering questions based on untenable presuppositions. For the principle we are violating in matters of that totalization is not that of faithfulness to the facts of existence, but that of keeping to conformity with the conditions of discursive meaningfulness. It is-to reemphasize - the linguistic proprieties that are being violated. And this perspective makes it possible to take a more narrowly targeted approach that is not committed to wholesale object dismissal in the manner of Russell's approach. We need not proscribe all talk about "all sets" or "all propositions" but rather must merely be careful about what we endeavor to say with these locutions." We need to embargo impredicative characterizations only in identificatory, definitional, and similarly item-specificatory contexts where ontological applications are at issue. Russell's wholesale rejection of self-inclusion-dismissing, for example, the selfinclusive contention "All propositions are either true or not" as meaninglesssimply slays the innocent along with the guilty.

In the final analysis, then, it is not the meaningfulness of impredicative characterizations but rather their employment in context of hypostatization that needs to be called into question, since a fallacy or illicitly presupposition is then unavoidable. For when the answer to the presuppositional question "Are the conditions for a meaningful item-identification met?" is No, then we cannot blindly barrel ahead as though it were Yes.

\section{A Kantian Postscript}

Immanuel Kant deserves to be acknowledged as the philosopher who, in his Critique of Pure Reason, first objected to illicit totalization. And he took this step because he saw it as a means for freeing himself from what he regarded as problematic and theoretically unwelcome entities. For as Kant saw it, such ideas as that of the "ultimate constituents of spatial regions" or "the ultimate causes of events" represent the products of illicit totalization. He rejects totalities that are not closed as it were and thereby impossible to survey in toto, since he held that experience is the key to our knowledge of existence, and of course we never experientially survey certain totalities as such. As he saw it, a fundamental fallacy is involved in such totalitarian conceptions:

The concept of totality is in this case [of the-world-as-a-whole] simply the representation of the completed synthesis of its parts; for we cannot obtain the concept from the apprehension of the whole-that being in this case impossible.... (CPuR, B456)

For Kant, such closure-defying, unsurveyable conceptions as that of the worldas-a-whole-one, whose content goes beyond the range of that which could ever be given in experience, is something ill-defined and thereby inappropriate. Only experiential interaction can assure actual existence-description alone can never do the job: 
[It is inappropriate to suppose] an absolute totality of a series that has no beginning or end [such as would be at issue with "the terminus of all successive divisions of a region" or "the initiation of all the causes of an event."] In its empirical meaning, the term "whole" is always only comparative. The absolute whole of quantity (the universe), the whole division [of a line segment], or of [causal] origination or of the condition of existence in general ... along with all questions as to whether this whole is brought about through finite synthesis or through a synthesis requiring infinite extension ... [are something altogether inappropriate]. (CPuR, A483-84 = B511-12)

With experiential unification unachievable, we can never appropriately reify such a totalitarian conception into that of an object that has a well-defined identity of its own.

For Kant, such improperly totalized items as "the-physical-world-as-a-whole" can never represent a well-defined object, but only an inherently fallacious conception that leads to inconsistency:

[As a sum-total of existence] the world does not exist in itself, independently of the regressive series of my representations, it exists in itself neither as an infinite whole nor as a finite whole. It exists only in the empirical regress of the series of appearances, and is not to be met with as something in itself. If, then, this series is always conditioned, and therefore can never be given as complete, the world is not an unconditioned whole, and does not exist as such a whole, either of infinite or of finite magnitude. (CPuR A503-05 $=$ B531-33)

Kant has it that the endorsement of such unsurveyably unbounded totalizations will result in untenable concepts that engender antinomies-actual logical selfcontradictions. For if we violate this proscription of ungrounded totalization trouble is bound to ensue by way of inconsistency:

If the world is a whole existing in itself, it is either finite or infinite. But both alternatives are false (as was shown in the [previous] proofs of the antithesis and thesis respectively). It is therefore also false that the world (the sum of all appearances) is a whole existing in itself. (CPuR, A506 = B534)

Kant insisted that illicitly totalization must fail to yield objects that have a welldefined identity and to accept such pseudo-objects as actual-to reify or hypostasize them-is to commit a fallacy that engenders the self-contradiction reflected in the antinomies that he enumerated and analyzed. As Kant saw it, the four classic "Antinomies" of Critique of Pure Reason exemplify the difficulty at issue. These Kantian Antinomies run as follows:

I. The totality of physical existence ( $=$ the world or the universe as a whole)

- is limited in the physical manifold of space and time;

- is unlimited in the physical manifold of space and time.

II. The ultimate simples of nature, the absolutely atomic, totally indecomposable constituents of physical substance 
- are pervasive throughout all of physical reality;

- are excluded from physical reality (i.e., do not exist as such).

III. The totality of natural occurrences (= the aggregate of events in physical realm)

- is of necessity determined by physical law;

- is free from determination by physical law.

IV. An absolutely necessary, self-engendering being-one that itself encompasses the totality of its own cause

- is physically or causally active in the natural world;

- is physically and causally excluded from the natural world.

In each case what we have at issue an extra-ordinary, somehow totalitarian (that is ultimate or absolute) object of consideration regarding which some far-reaching feature is both flatly affirmed and categorically denied. What is being claimed throughout is (structurally speaking) that a certain putative object $(A)$ both does and does not have a certain physically geared feature $(F)$. Conflicting predications are at issue: " $A$ is $F$ " and " $A$ is not- $F$." And, so Kant maintained, equally good arguments can be constructed either way, so that both the thesis and the antithesis can be rendered plausible.

On this basis, Kant's antinomies are by him seen as engendering a paradox along the following general structural lines:

(1) Item $A$ (e.g., physical-existence-as-a-whole) is a legitimate object of prediction.

(2) There is cogent reason for saying that item $A$ (e.g., physical existence) is $F$ (e.g., limited). (A substantiable fact grounded in argumentation that Kant presents in some detail.)

(3) There is cogent reason for saying that item $A$ (e.g., physical existence) is not $F$ (e.g., unlimited). (Also a substantiable fact rooted in presented argumentation.)

(4) (2) and (3) are logically incompatible if (and whenever) $A$ exists as a legitimate object of predication. (A fact of logic.)

(5) Therefore, by (2)-(4) item $A$ does not exist because it is a fact of logic that no legitimate object of predication can have incompatible predicates.

(6) (5) contradicts (1).

As Kant saw it, the natural place to break this chain of inconsistency is at thesis (1) which-so he maintains-is the weak spot of the antinomy. For him the proper lesson to be drawn is that identifiable particulars can exist as proper objects of predication whereas abstract totalities are vitiatingly problematic in this regard.

The four Kantian antinomies arise from a series of corresponding questions of a common format: 
Is the ultimate unit of $[i]$ limited or unlimited in point of $[i i]$ ? where the four cases stand as follows:

\section{[i]}

1. physical existence

2. physical divisibility

3. natural occurrence

4. existential selfsufficiency [ii]

spatio-temporal distribution

physical diffusion

lawful determination

causal participation in

nature

And all of these questions make the common supposition that that ultimate item at issue in $[i]$ actually exists--something that Kant emphatically denies.

Kant thus argues that in each and every case, the resolution of the antinomy consists in rectifying a mistaken presupposition that ultimate item $A$ at issue is a bona fide (physical) existent that is capable of being a proper bearer of (physically oriented) predicates such as spatiotemporal extent, physical existence, lawful determination, or causal efficacy. Such ultimates are communicative devices for thought and expression but not items of natural existence of which physical features can be predicated. (This is why the second, negative side of the antinomies always comes closer to the mark.) Kant's position is not so much that of Nicholas of Cusa that certain totalities are beyond human comprehension, as that they are not defined as identified individuals upon which the machinery of subject/predicate logic can get a purchase.

Kant's position here is certainly discussible. One could, for example, contend that those supposed "cogent reasons" at issue in the aporetic theses (2)-(3) constitute the vulnerable presupposition of the argument rather than thesis (1). Or again one could try to dissolve the contradictions on grounds of equivocation, arguing (for example) that the sense in which the physical world is limited in space-time is different from the sense in which it is not so limited.

All the same, Kant's idea of rejecting certain totalities as illicit is not all that implausible. No painting of a painting can represent all of its detail. And even so no thought or theory can encompass the entire reality to which it itself belongs. The hypothetical projection of such an item envisions a totality that is incapable of realization-one whose acceptance conflicts with the things we know about the nature of human knowledge. As Kant saw it, the synoptic (totalitarian) preview of the claims of classical metaphysics is simply unavailable to us humans. We humans have to operate within the world and therefore from a particular (specifically human) perspective. We have no cognition-external Archimedean fulcrum outside the real of our thought from which to move the entire domain of thought as a whole.

And this Kantian perspective relates to the relationship between inner and outer. We humans are emplaced within nature and by this very fact are limited as to the sort of information we can obtain about nature. All of our observation-based judg- 
ments are partial and thereby incomplete in scope. Every observer has a body of information whose limitedness precludes anything like an all-inclusive view of things. As far as actual knowledge goes, totalities are off limits-be it the totality of actual experience (the self) or of its objects (the world) or of its rationale (ultimate purpose or God)..$^{12}$ Such ideas represent useful mind-contrivances for the development of actual knowledge but do not - cannot-themselves constitute actual objects of knowledge: as Kant saw it, they are not entities at all, but only practical resources, instrumentalities of cognitive procedure useful only for contrasting what we actually have with what we would ideally like. Those improperly totalized items simply do not exist as such.

And so, as Kant saw it, those improperly totalized items are not entities at all, but only practical resources, instrumentalities of cognitive procedure useful only for contrasting what we actually have with what we would ideally like. And paradox accordingly results because here the presupposition of existence is not satisfied. There is, accordingly, a substantial analogy between Kant's position and the line of thought set out above in relation to Russell's approach.

However, another aspect of the matter must be noted. Kant thought that by representing such items as "the world" or "the deity" as illicit totalizations he could eliminate the salient issues of traditional ("pre-critical") metaphysics. In particular, his critique of inappropriate totalization was an integral part of his dismissal of the cosmological argument for the existence of an all-creative God to serve as producer of the world. As he saw it, if there is no such thing as "reality-at-large" or "the world as a whole," then the issue of its causal origination is rendered moot subject to the plausible principle that: Only an item already known to exist needs to have its existence explained.

Now although all this may seem sensible enough, it is nevertheless questionable whether this line of argumentation achieves the overall result that Kant intended for it. For while his principle that "Only existing entities stand in need of an existence-explanation" is all very well as far asit goes, it does not really manage to negate the potential utility of reasoning in the manner of the cosmological argument. The proscription of world-totalization may blank "Why does the world-asa-whole exist?" But it does not affect-let alone invalidate-the ontological question in the form originally envisioned by Leibniz: "Why is there something rather than nothing?" And this condition of affairs prevails more widely. Those metaphysical issues which Kant sought to eliminate by rejecting of totalized objects can generally be reconstituted in ways that avert the illicit reification that is supposedly at issue. But while Kant's prohibition of unrestricted totalization may not in the end prove to have been quite as powerful a destroyer of metaphysical issues as he thought. It is and will ever remain a philosophical resource of substantial utility. ${ }^{13}$ 


\section{Notes}

To be sure, where the matter is one of picking out items from a pre-established group, there need be no difficulty.

2 On problems of self-reference, see R.M. Sainsbury, Paradoxes (Cambridge: Cambridge University Press, 1987, 2nd ed. 1995), pp. 121-122 of the second edition.

${ }^{3}$ What is at stake here is a flaw different from the more familiar identification-defect that arises where there is vagueness-a penumbrally "fuzzy" whole where one cannot tell exactly what is within and what is not. For in our present case, the very item at issue is not defined as a meanignful unit because it itself is presupposed by its mode of indentification/specification. Accordingly it is not the purported totality's membership but its very identity that is questionable.

${ }^{4}$ Nicholas of Cusa, Of Learned Ignorance, tr. by G. Heron (New Haven: Yale University Press, 1954), Bk. III, Chap. I.

"Troughout these formulations, "all" is to be construed as "all but only." Accordingly, a supertruth (for example) would be said to be a truth that conjoins all members of $\{p: p$ is true $\}$, the set of all propositions $p$ such that $p$ is true.

- This paradox-often called The Barber of Seville Paradox - is an informal variant of Russell's Paradox in set theory (for which, see pp. $\$ 3-55$ below). See T.S. Champlin, Reflexive Paradoxes (London: Routledge, 1988), esp. pp. 172-174.

3 this ITP principle might well be grounded in a broader principle to the effect that fore successful identification the items being referred to in the course of the process must themselves already have been identified. This approach, in effect, seems to have lain at the basis of Henri Pointcare's treatment of the paradoxes, a s trategy whose decided difference from Russell's neither he nor Russell seem to have appreciated sufficiently. See the lucid discussion of all these matters in Charles S. Chihara's Ontology and the Vicious Circle Principle (Ithaca, NY: Cornell University Press, 1973), esp. pp. 138-140.

${ }^{8}$ See Bertrand Russell and A.N. Whitehead, Principia Mathematica, Vol. 1 (Cambridge: Cambridge University Press, 1910) [re-issued in paperback in 1967], pp. 31, 37. Elsewhere Russell puts it as follows: "If provided a certain collection has a total, it would have members only definable in terms of that total, then the said collection has not total." American Journal of Mathematics, vol. 30 (1908), pp. 222-262 (see p. 240). Russell went on to explain that "When I say that a collection has no total, I mean that statements about all its members are nonsense." (Ibid,; see also P.M.) He appears to think, however, that the culprit here is "all" instead of "it"?

${ }^{9}$ Bertrand Russell, "Matematical Logic as Based on the Theory of Types" (1908), reprinted in J. van Heijenoort, From Frege to Gödel (Cambridge, MA: Harvard University Press, 1967), pp. 153-182; see p. 155. Elsewhere, Russell is more cautious (see note 8, above). See also P.M., Vol. 1, pp. 31, 36 . The phrase "only definable" helps matters but also introduces problems of its own becasue that "only" is somewhere between difficult and inappropriate to cash in.

"Thus it is necessary, if we are not to sin against the above negative principle, to construct our logic without mentioning such things as 'all propositions' or 'all properties'--and without ever having to say that we are excluding such things.

"We thus need not follow Russell in rejecting an ontology of classes and adopting a "no class theory" on grounds that statements about "all classes" are meaningless. There lies open the option of holding, with Godel, that classes can be abstract objects that "exist independently of our definitions and constructions." For we can merely hold that certain ways of trying to refer to classes are flawed and cannot succeed in effecting such reference. Compare Kurt Godel, "Russell's Matematical Logic," in the Philosophy of Bertrand Russell, ed., by P. Schilpp (New York: Tudor, 1969)., pp. 123153

${ }^{12}$ See the discussion of these ideas at CPuR A689-B708 $=$ A689-B717.

"I am grateful to Alexander Pruss for constructive comments.

Nicholas Rescher, University Professor of Philosophy, University of Pittsburgh Department of Philosophy, 1012 Cathedral of Learning Pittsburgh, Pennsylvania 15260 U.S.A. rescher@vms.cis.pitt.edu 